\title{
VORWORT ZUR ERSTEN RUSSISCHEN AUFLAGE
}

Ein Vorwort wird oft geschrieben, weil der Autor die Absicht hat, die kommenden Schläge der Kritik durch eine geschickte Verteidigung zu parieren. Meist ist dabei das offene Bekenntnis der Mängel der eigenen Arbeit mit dem Hinweis auf ihre absolute Unvermeidbarkeit verbunden. Eine ähnliche Form der literarischen Selbstverteidigung ist besonders dann nötig, wenn der Gegenstand der Betrachtung ein sich schnell entwickelnder Zweig der Wissenschaft oder Technik ist, der sich im ersten Stadium seines Werdens befindet. Eine solche Situation ist geradezu charakteristisch für den gegenwärtigen Stand der Kernfusionsuntersuchungen. Deshalb muß der Autor dieses Buch mit Selbstkritik beginnen.

Vor allem muß er den Leser im voraus darauf aufmerksam machen, daß der Titel des Buches seinen Inhalt nicht genau bezeichnet. Der Titel weist zwar auf das Ziel hin, das wir anstreben; dieses Ziel leuchtet aber kaum erst am fernen Horizont auf, und zu ihm führt nur ein langer und beschwerlicher Weg. In diesem Buche kann natürlich nur derjenige kleine Abschnitt dieses Weges beschrieben werden, der bis jetzt durchlaufen wurde. Man erhält so eine Vorstellung davon, welche Schwierigkeiten bis zur Lösung der gestellten Aufgabe noch überwunden werden müssen. Der Leser soll hier keine Berechnungen und Konstruktionen suchen, die sich auf Projekte technischer thermonuklearer Reaktoren bezieken. Sie existieren noch nicht, denn wir befinden uns jetzt erst auf einer Vorstufe, auf der die wissenschaftlichen Grundlagen der künftigen Kernfusionstechnik entstehen.

Als Grundlage der thermonuklearen Forschung muß eine neue, künstlich erzeugte Erscheinungsform der Materie, das Hochtemperaturplasma, dienen.

Im Buche werden daher zunächst die wesentlichen theoretischen Überlegungen behandelt, die sich auf die im Plasma ablaufenden Prozesse beziehen. Es werden dann Aufheizungs- und Wärmeisolierungsmethoden betrachtet. Die konkreten Anlagen, die zur Durchführung von Experimenten an Hochtemperaturplasmen gebaut wurden, werden beschrieben; und schließlich werden die Resultate dieser Versuche kritisch eingeschätzt.

In den ersten vier Kapiteln wird die Physik des Plasmas behandelt. Die übrigen vier Kapitel bringen eine Analyse der Hauptrichtungen der experimentellen Kernfusionsforschung.

Auswahl und Verteilung des Stoffes mußte so erfolgen, daß man zu einem klaren Verständnis der Probleme kommt. Deshalb wurde davon abgesehen, ein dürftiges Skelett experimenteller Fakten mit einer schwierigen mathematischen 
Einkleidung zu versehen. Es wurde vielmehr angestrebt, ein vernünftiges Verhältnis zwischen theoretischer und experimenteller Information einzuhalten. Im Buch wird deshalb im wesentlichen nur ein Minimum an theoretischen Kenntnissen geboten, welches zur allgemeinen Orientierung in der Physik des Hochtemperaturplasmas nötig ist. Umfangreiche Schlußfolgerungen aus den Formeln wurden vermieden. In allen Fällen, wo man entweder die Anschaulichkeit oder die Strenge opfern muß, erhält die Anschaulichkeit den Vorrang.

Das im Buch ausgewertete Material wurde mit wenigen Ausnahmen Arbeiten entnommen, die bis Ende 1962 fertiggestellt waren. Bei allen Berechnungen wird in der Regel das Gausssche CGS-System verwendet.

Der Autor spricht dem großen Kollektiv der Mitarbeiter der Abteilung für Plasmaforschung im Kuntschatow-Institut für Atomenergie der Akademie der Wissenschaften der UdSSR seinen aufrichtigen Dank aus für die äußerst wertvolle Mitarbeit bei der Vorbereitung des Buches, für die Überlassung experimenteller Daten, Berechnungen, graphischer Darstellungen und Aufnahmen und auch für viele wertvolle Ratschläge und Bemerkungen.

Besonders erwähnt sei die Mitarbeit von R. S. SAGDEJEw. Er bearbeitete $\S 21$ und den größten Teil von $\S \mathbf{4 5}$.

Der Autor dankt außerdem W. M. Glagolew für die Ausarbeitung des Abschnittes über Hochfrequenzhalterung des Plasmas ( $\$ 63$ und 64). Ohne die Mitarbeit von E. W. ARTJuschkow hätte das Buch wahrscheinlich nicht erscheinen können. Er hat mit großer Gewissenhaftigkeit und Sachkenntnis die schwierige Arbeit der Vorbereitung des Manuskriptes zum Druck übernommen. Außerdem hat er eine Reihe schwieriger und zeitraubender Rechnungen durchgeführt und eine große Anzahl der im Buch enthaltenen graphischen Darstellungen gezeichnet.

L. A. Arzimowrtsch 